\title{
A new magnetotelluric monitoring network operating in Agri Valley (Southern Italy): study of stability of apparent resistivity estimates
}

\author{
Marianna Balasco $\left({ }^{1}\right)$, Vincenzo Lapenna $\left({ }^{1}\right)$, Gerardo Romano $\left({ }^{1}\right)$, \\ Agata Siniscalchi $\left({ }^{2}\right)$ and Luciano Telesca $\left({ }^{1}\right)$ \\ (') Istituto di Metodologie per l'Analisi Ambientale, CNR, Tito Scalo (PZ), Italy, \\ ${ }^{(2)}$ Dipartimento di Geologia e Geofisica, Università degli Studi di Bari, Italy
}

\begin{abstract}
Variations detected in geophysical, especially electromagnetic, parameters in seismic active areas have been sometimes attributed to modifications of the stress field. Among the different geophysical methods, magnetotellurics (MT) could be one of the most effective because it allows us to explore down to seismogenic depths. Continuous MT recording could allow us to evaluate whether possible variations are significantly correlated with the seismic activity of investigated area. To assess the significance of such observations we must be able to say how well an apparent resistivity curve should be reproduced when measurements are repeated at a later time. To do this properly it is essential to know that the estimated error bars accurately represent the true uncertainties in comparing the transfer functions. In this work we will show the preliminary results obtained from the analysis of the data coming from the new MT monitoring network installed in Agri Valley. This analysis gives us the possibility: i) to better study the temporal stability of the signals, ii) to better discriminate the noise affecting the measures by remote reference estimation. The performed analysis disclosed a relatively low degree of noise in the investigated area, which is a promising condition for monitoring.
\end{abstract}

Key words magnetotelluric - remote reference apparent resistivity curve - earthquake precursors

\section{Introduction}

A great variety of phenomena such as changes in electric (Huang and Liu, 2006), magnetic and electromagnetic fields and/or changes in the electric structure of subsoil (Nagao et al. 2002; Zhao and Qian, 1994) have

Mailing address: Dr. Luciano Telesca, Istituto di Metodologie per l'Analisi Ambientale, CNR, C. da S.Loja 5, 85050 Tito (PZ), Italy; e-mail: luciano.telesca@imaa.cnr.it been associated to seismic activity (Park et al. 1993; Merzer et al. 1997; Karakelian et al. 2002). The magnetotelluric (MT) method is a geophysical technique used to image the subsurface electrical resistivity (Cagniard, 1953) using as a source the Earth's natural electromagnetic field, whose range of periods is very broad. Under the assumption that the external sources are spatially uniform and neglecting the displacement current, the physical problem may be treated as one in pure diffusion (Keller and Fischknecht, 1966). The penetration depth of the electromagnetic field increases with period and resistivity and can reach several tens of kilometers for longer periods, depending on the resistivity of the rocks. By inverting the impedance tensor, which linearly relates the two hor- 
izontal electric components to the horizontal magnetic ones, the electrical resistivity distribution of the Earth's interior can be defined.

The use of MT data as a seismic precursor can be limited by problems related to the reliability of MT results. These problems are connected with the sources of electromagnetic field, the complexity of the observed system (the Earth) and the need to integrate the MT data with other geophysical data not always available. Violation of plane wave assumption due to cultural electromagnetic noise (Egbert and Booker 1986) or variation of MT response produced by conductivity anomalies in the shallow layers of subsoil (Bahr,1988) can hide the variations of ground resistivity linked to the seismic activity.

In 2003 a magnetotelluric (MT) monitoring station was installed by the Institute of Methodologies for the Environmental Analysis (IMAA) at Barricelle in Agri Valley, Southern Italy (Balasco et al. 2004a). This area is very interesting for its structural geologic context (Giano et al. 2000), its recent and historical seismicity and also for the presence of numerous oil wells that expose it to a high environmental risk. During three years of observation (2003-2005) there were no significant seismic events, and, therefore, it was possible to define the characteristic MT curve of the site as function of period for which we studied the stability of the apparent resistivity and the fluctuation dynamics of the apparent resistivity values at different time scales (Balasco et al. 2007). Because of unavailability of Barricelle site, the station was removed and in 2006 the IMAA installed MT stations at other two locations in the Agri Valley, near the principal faults of this area.

The increased number of MT stations gives the possibility to improve the estimates of apparent resistivities by remote reference method in which horizontal magnetic fields recorded simultaneously at a second remote site are correlated with the electromagnetic fields at local site: this method provides a clear identification of coherent noise in contaminated MT data.

Our aim is to obtain a good background for the future analysis characterizing the sites where the MT stations are installed. This could be useful to analyse resistivity variations ob- served during the monitoring. Furthermore, in this work, we carry out the analysis of the stability of the resistivity estimates to choose a «time window» which can represent a good compromise between reliability and sensitivity of our data.

\section{The magnetotelluric method and data analysis}

A classical MT sounding uses the measurements of electromagnetic fields induced in the Earth by variations in the external geomagnetic field to obtain, by means a frequency-dependent impedance tensor $|Z|$, the apparent resistivity. The relationship between two orthogonal component of electric $\bar{E}$ and magnetic $\bar{M}$ fields is given by the following equation (Kaufman and Keller, 1981)

$$
\begin{aligned}
& E_{x}(\omega) \quad Z_{x x}(\omega) Z_{x y}(\omega) H_{x}(\omega) \\
& E_{y}(\omega)=Z_{y x}(\omega) Z_{y y}(\omega) H_{y}(\omega)
\end{aligned}
$$

where $\omega$ is the angular frequency, and $\left(E_{x}, E_{y}\right)$ and $\left(H_{x}, H_{y}\right)$ represent respectively the electric and magnetic components in an orthogonal reference. As a simple linear system, the transfer function $Z(\omega)$ acts as a filter, while the magnetic and electric fields represent the input and output respectively.

The apparent resistivity $(\rho)$ of the ground is defined by the following equation:

$$
\rho_{i j}^{a}(\omega)=\frac{1}{\mu_{o} \omega}\left|Z_{i j}(\omega)\right|^{2}
$$

where $\mu_{0}$ is the permeability of the vacuum and $Z_{i j}$ are the complex components of the tensor defined in eq. (2.1), with $i, j=x$ or $y$.

At the moment, in Agri Valley (fig. 1) two MT monitoring stations are working: MN station, located in Marsico Nuovo (lat. 40.42533 long. 15.72950) and TR station, located in Cozzo La Gatta (lat. 40.29668 long. 15.80554), away from urban and industrial centers. The MT monitoring stations are equipped with a receiver MT24LF (Magnetotelluric 24-bit A/D Low Frequency system) and two induction coils (EMI Inc., BF4) which record the magnet- 


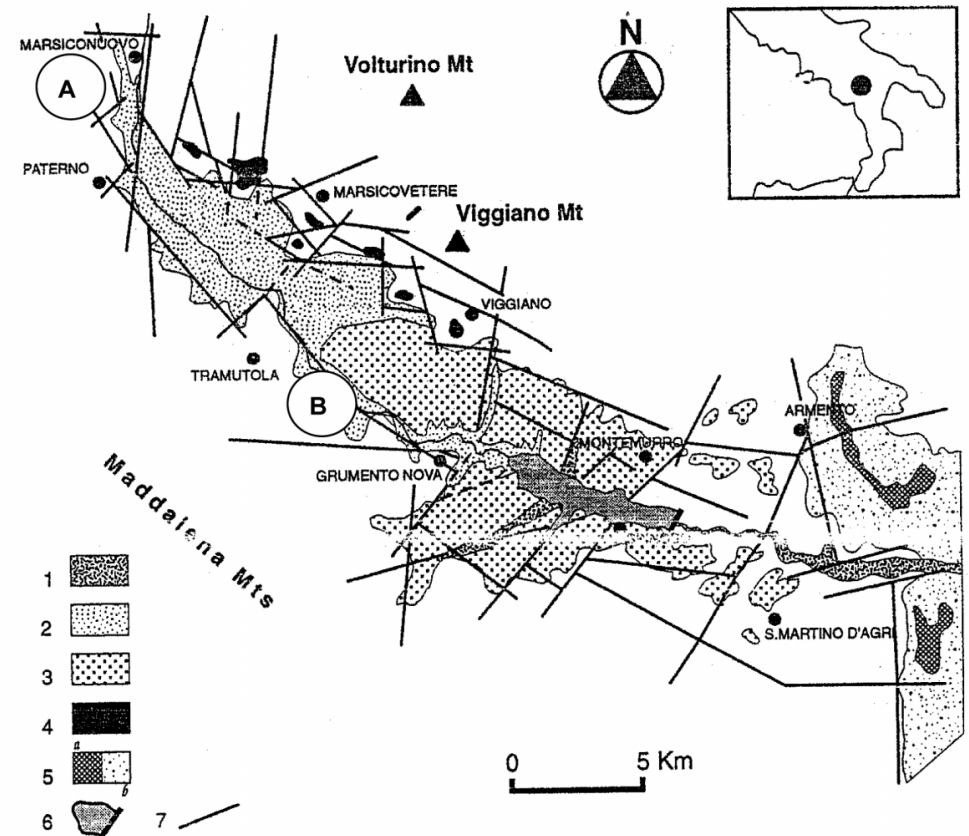

Fig. 1. Structural geological context of the investigated area. The alluvial deposits are shown: recent (1), Holocene-upper Pleistocene (2), upper-middle Pleistocene (3); subaerial slope deposits, upper-middle Pleistocene (4); conglomerates and sands, middle-lower Pleistocene (5); Pertusillo Dam (6); faults (7) (Giano et al., 2000). A: location of the magnetotelluric monitoring station in Marsico Nuovo. B: location of the magnetotelluric monitoring station in Tramutola

ic fields. MN station measures two components of the magnetic field along $0^{\circ} \mathrm{N}$ and $90^{\circ} \mathrm{N}$ directions ( $H_{x}$ and $H_{y}$ respectively) and the two components of the electrical field in the same directions $\left(E_{x}\right.$ and $\left.E_{y}\right)$ by means of $20 \mathrm{~m}$ long electrical dipoles. TR station measures the same components of the magnetic field as the MN station, but also measures the electric field by means of three electrical dipoles (50 m length): $E_{x}$ along $0^{\circ} \mathrm{N}$ direction and $E_{y 1}$ and $E_{y 2}$ in $90^{\circ} \mathrm{N}$ direction.

In this study we have analyzed two data sets of apparent resistivity, obtained by a magnetotelluric recording at $\mathrm{MN}$ and $\mathrm{TR}$ sites from July to November, 2006. The frequency of data recording was set to $6.25 \mathrm{~Hz}$ in continuous mode and about every five days we acquired data at high frequency sampling $(500 \mathrm{~Hz})$ for 30 minutes at 1:00 GMT choosing this window time to take advantage of the better signal to noise ratio (Garcia and Jones, 2002). The ap- parent resistivity curves $\rho_{x y}$ and $\rho_{y x}$, related respectively to the off-diagonal components of impedance tensor $Z_{x y}$ and $Z_{y x}$, were estimated using the procedure and the Robust Transfer Function Estimation Program for data reduction described in (Egbert, 1997).

The continuous data stream was divided into subsets of 45000, 90000, 135000 and 180000 values, corresponding to intervals of 2, 4, 6 and $8 \mathrm{~h}$ respectively and processed individually. This segmentation was devised to have a sufficiently long sample for reliable statistics (Eisel and Egbert, 2001). The procedure was applied to all the MN and TR subsets.

The estimation of the apparent resistivity curve was obtained with a stability analysis and a consistency analysis. The stability was performed using the median value $\left(\rho_{\text {med }}\right)$ for each subset, because it is unaffected by outliers (Balasco et al. 2004b). The consistency of $\rho_{\text {med }}$ 
was performed by the calculation of $\rho_{\text {Wmed }}$ (weighted median) for each frequency $(\omega)$ given by

$$
\begin{aligned}
& \sum_{i: \rho_{i}<\rho_{\text {Wmed }}} \frac{p_{i}}{\sum_{i: 1, n} p_{i}}<\frac{1}{2} \\
& \sum_{i: \rho_{i} \geq \rho_{\text {Wrmed }}} \frac{p_{i}}{\sum_{i: 1, n} p_{i}} \geq \frac{1}{2}
\end{aligned}
$$

with weights pi determined for each subset $i$. In our case

$$
\left\{\begin{array}{l}
\zeta_{i} \leq 2 \longrightarrow p_{i}=1 \\
2<\zeta_{i} \leq 99 \longrightarrow p_{i}=1-\frac{\zeta_{i}}{100} \\
\zeta_{i}>99 \longrightarrow p_{i}=0.001
\end{array}\right.
$$
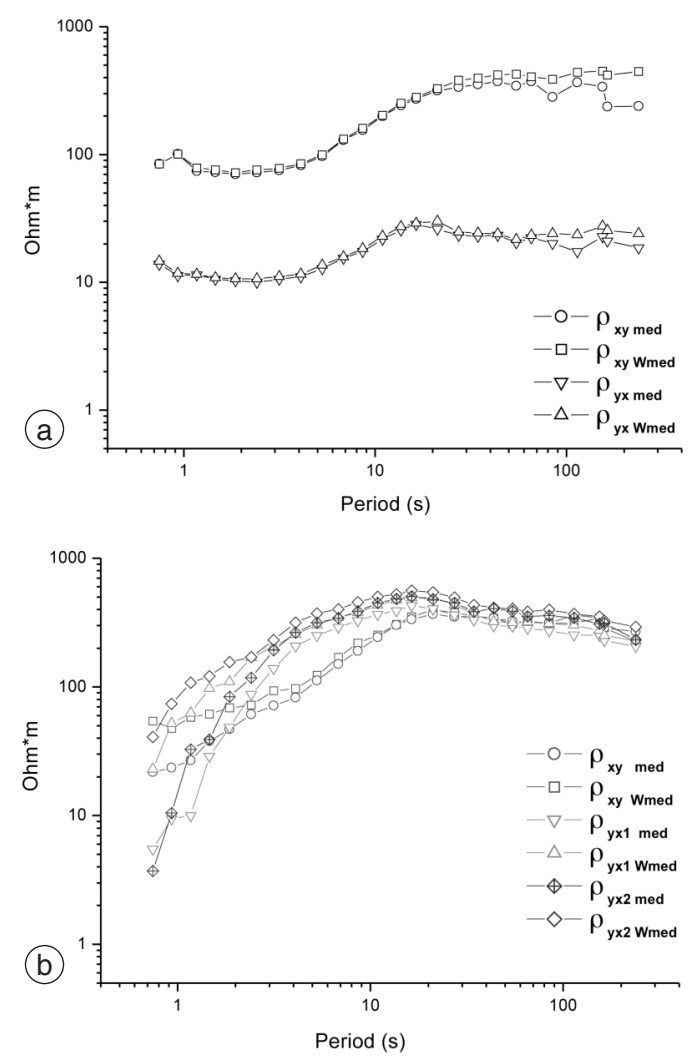

Fig. 2a,b. Comparison between $\rho_{\text {med }}$ and $\rho_{\text {Wmed }}$ curves for (a) the MN site and (b) the TR site.
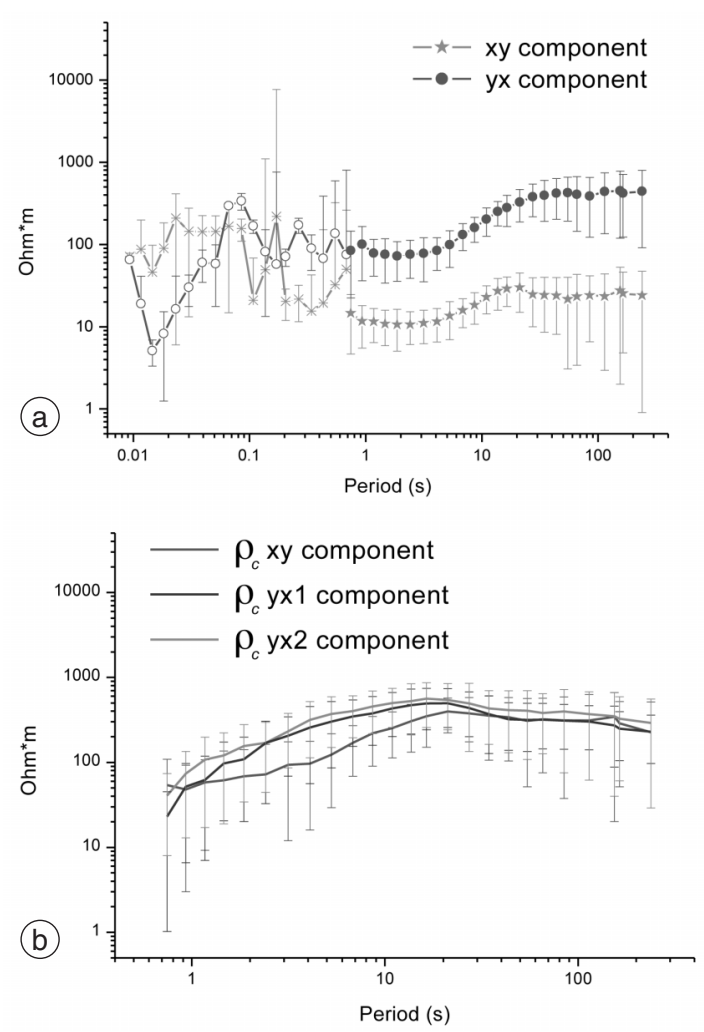

Fig. 3a,b. a) Characteristic apparent resistivity curve and error bars in high and low frequency band relative to $\mathrm{MN}$ site; b) characteristic apparent resistivity curve and error bars low frequency band relative to TR site

where $\zeta_{i}$ is $\left(\left(\sigma_{i} / \rho_{i}\right) * 100\right)$ and $\sigma_{i}$ is the standard deviation associated to each estimate $\rho_{i}$ of apparent resistivity.

Figure 2a,b shows the comparison between the $\rho_{\text {med }}$ and $\rho_{\text {Wmed }}$ curves of the MN (fig. 2a) and TR sites (fig. 2b). These curves were obtained using the $8 \mathrm{~h}$ which compared with 2,4 and $6 \mathrm{~h}$ subsets result more stable. The $\rho_{\text {Wmed }}$ curve was selected as the characteristic apparent resistivity curve $\left(\rho_{C}\right)$, and used as reference in the further analyses. In fact, it is smoother than $\rho_{\text {med }}$ curve and weakens the dead band effect highlighted by anomalous slope. 

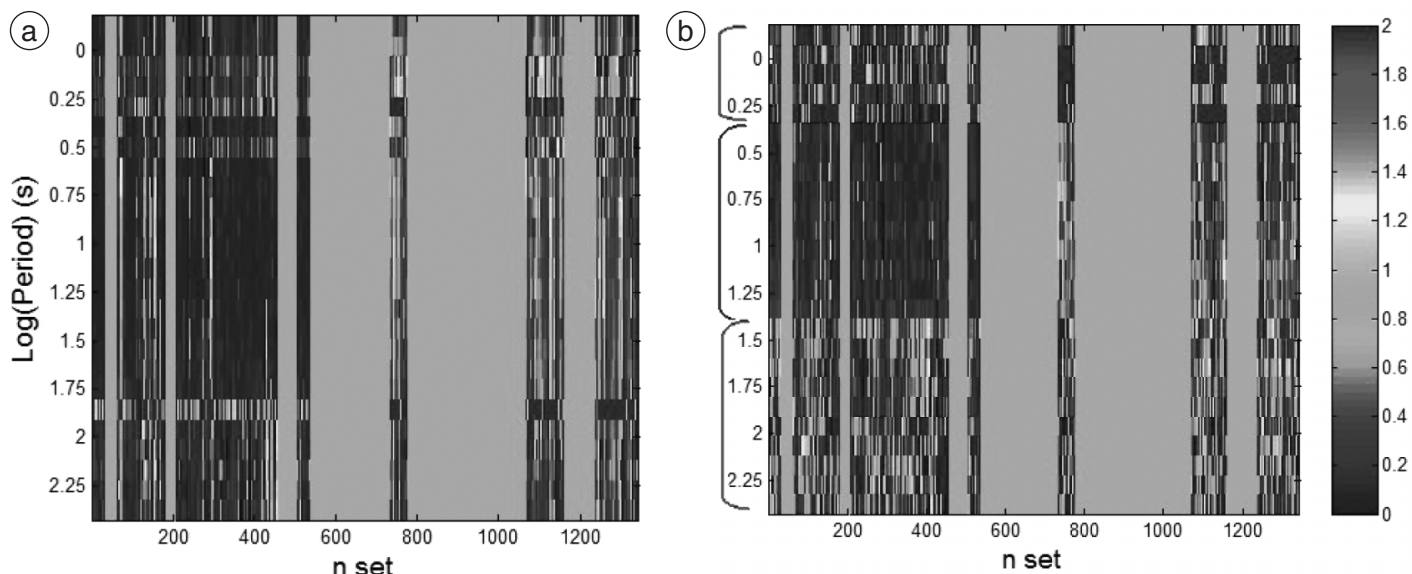

Fig. 4a,b. Variogram for MN site: a) $x y$ component; b) $y x$ component; the vertical labels on the left highlight the high variability zones (red labels $[0.74 \mathrm{~s}, 1.86 \mathrm{~s}]$ and $[27.3 \mathrm{~s}, 238.3 \mathrm{~s}]$ ) and the stable zone (blue label [2.40s, 21.14s]).
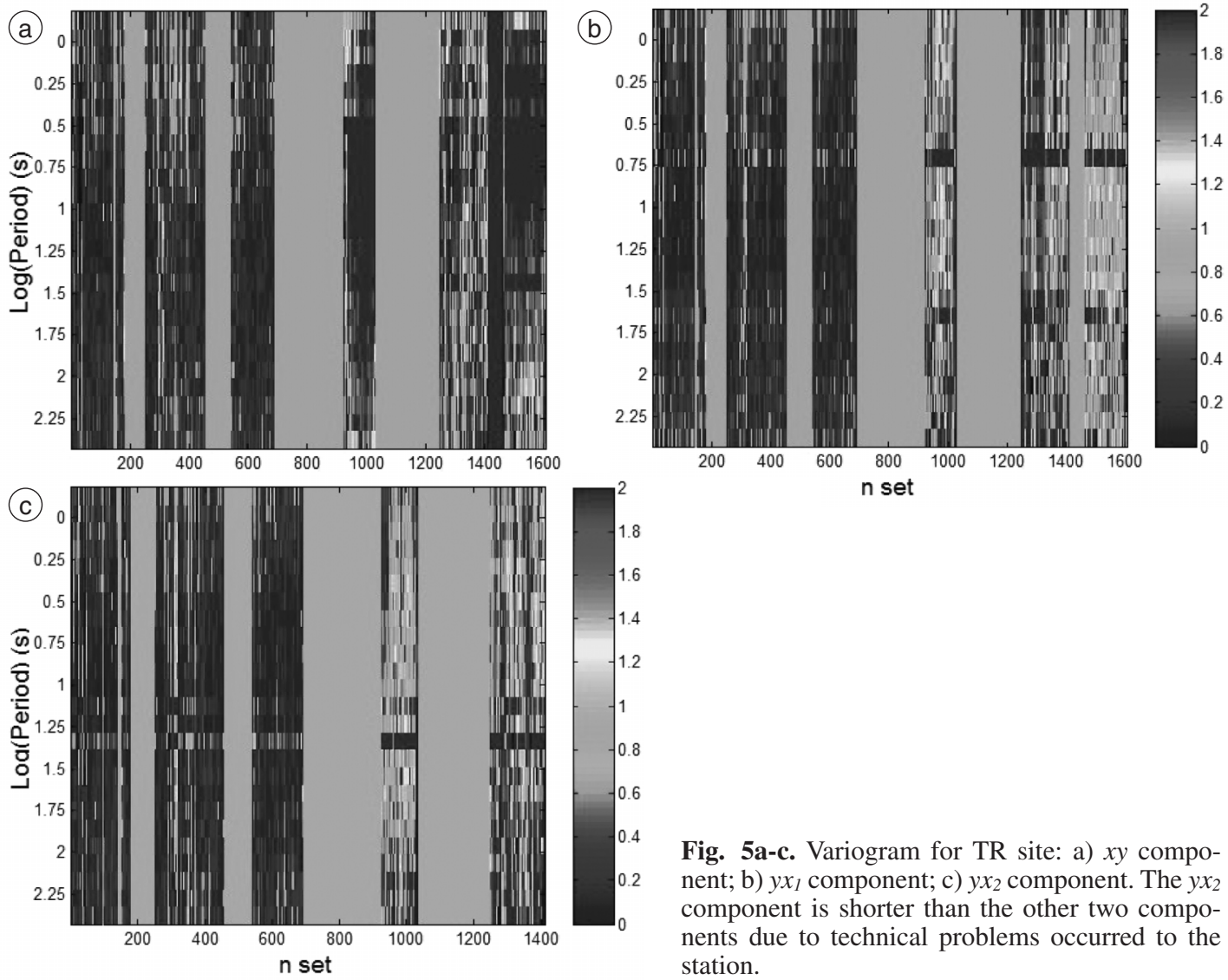

Fig. 5a-c. Variogram for TR site: a) $x y$ component; b) $y x_{1}$ component; c) $y x_{2}$ component. The $y x_{2}$ component is shorter than the other two components due to technical problems occurred to the station. 

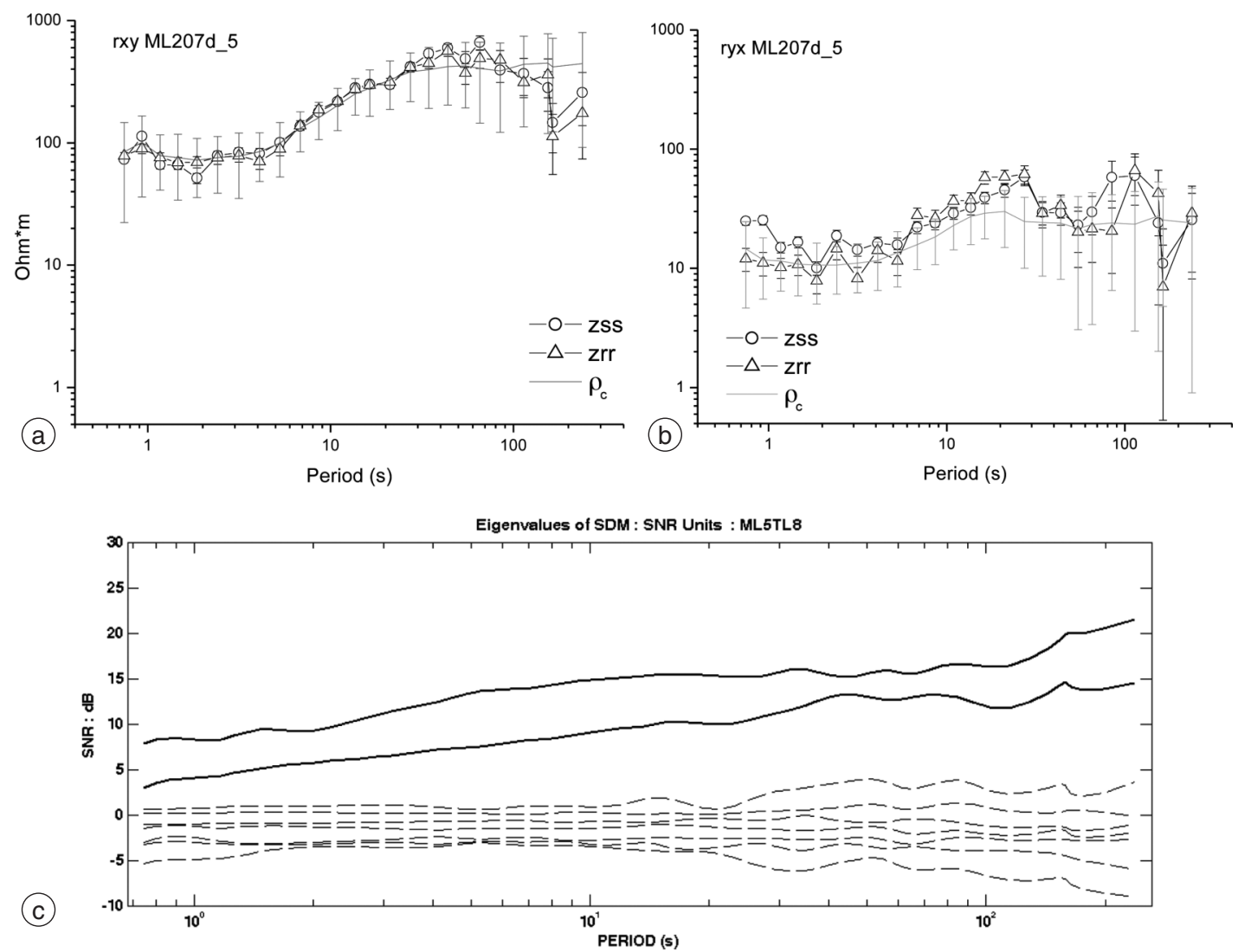

Fig. 6a-c. Comparison between the apparent resistivity curves estimate for the MN site computed with robust single station method ( $z s s$ curve), using TR site as a reference ( $z r r$ curve) for $x y$ component (a) and $y x$ component (b). (c) Eigenvalues of SDM for MN site: the two dominant eigenvalues are plotted with solid line.

The $\rho_{C}$ curves with error bars defined by $\Delta \rho_{c}(\omega)=1 / n \sum_{i=1, n}\left|\rho_{c}(\omega)-\rho_{i}(\omega)\right|$, are shown in fig. 3a,b. For MN site (fig. 3a) the curve shown is obtained joining together the $\rho_{C}$ Low Frequency (LF) curve $[0.744 \mathrm{~s}, 238.312 \mathrm{~s}]$ and the $\rho$ High Frequency (HF) curve [0.009 s, 0.682 s]. Even if the HF curve, obtained from a poor statistical sample, is characterized by large error bars and by a scattered behaviour, it is shown because it highlights the period range in which the static shift originated.

To study the behaviour of the apparent resistivity variations and not knowing their «temporal scale» we calculated the variograms for all the two-hour subsets by means of the formula

$$
\Delta \rho_{i}(\omega)=\left|\frac{\log \rho_{i}(\omega)-\log \rho_{c}(\omega)}{\log \left(\rho_{c}(\omega)\right)}\right|
$$

The variability of the apparent resistivity $\rho_{i}$ from the characteristic resistivity $\rho_{C}$, for all the two-hour subsets in period range (0.74-238.31 s), for both sites is shown in fig. 4 and fig. 5 . The monochromatic bands correspond to data missing.

In those figures, all the good estimates are associated with values of $\Delta \rho_{i}(\omega)<1$ (light green in the colour scale). Taking this into account, 
we can assess that estimates related to $\mathrm{MN}$ site show a general high degree of stability. In particular, $x y$ component is more stable than $y x$ component where a great variability can be observed at high and low period ranges and in particular at some fixed periods. Regarding TR site, instead, the variograms show a twofold behaviour: a good degree of stability for the first subsets, and an increasing instability in the last subsets, probably due to equipment technical problems. Comparing the MN and TR variograms, we selected two sets, having the same start time, in which $\Delta \rho_{i}(\omega)$ is shortest. Two different MT processing schemes were applied to the data: a robust single site processing scheme (zss) and a remote reference (zrr, Gamble et al., 1979; Egbert and Booker 1986) processing scheme. The use of the remote reference method removes uncorrelated noise from MT data. In this way we can better understand the character of the signal and noise in MT data. Figure $6 a, b$ shows the apparent resistivity curves estimate for the MN site with the two above-mentioned methods compared with the characteristic apparent resistivity curve. In $z r r$ estimates, we use TR site as a reference. For $x y$ component, the two methodologies give quite similar results (past 10s the three curves in fig. $6 \mathrm{a}$ are fairly coincident) while for $y x$ component we have dissimilarities at high and low pe-
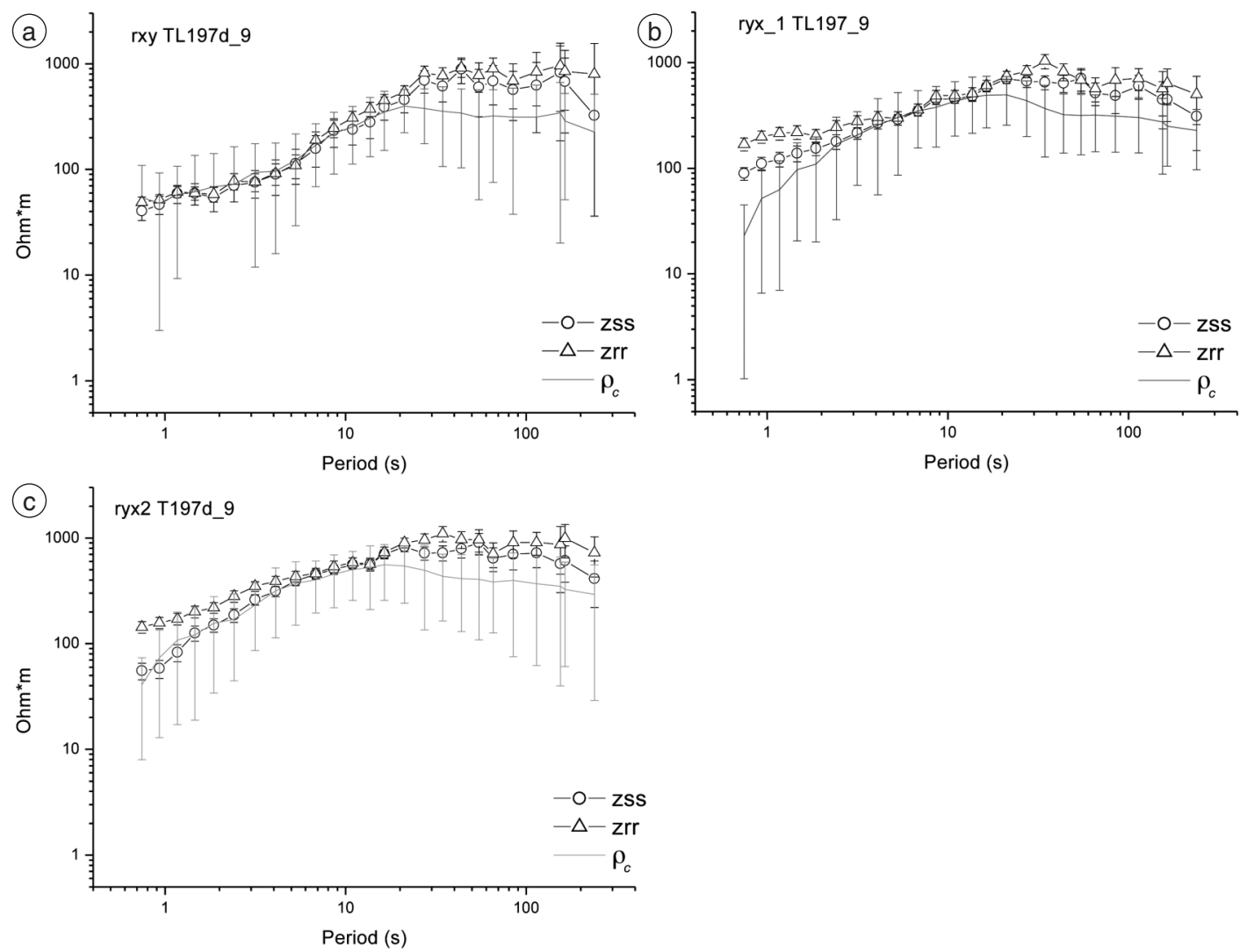

Fig. 7a-c. Comparison between the apparent resistivity curves estimate for the TR site computed with a robust single station method ( $z s s$ curve), using MN site as a reference ( $z r r$ curve) for $x y$ component (a), $y x_{l}$ component (b), $y x_{2}$ component (c). 


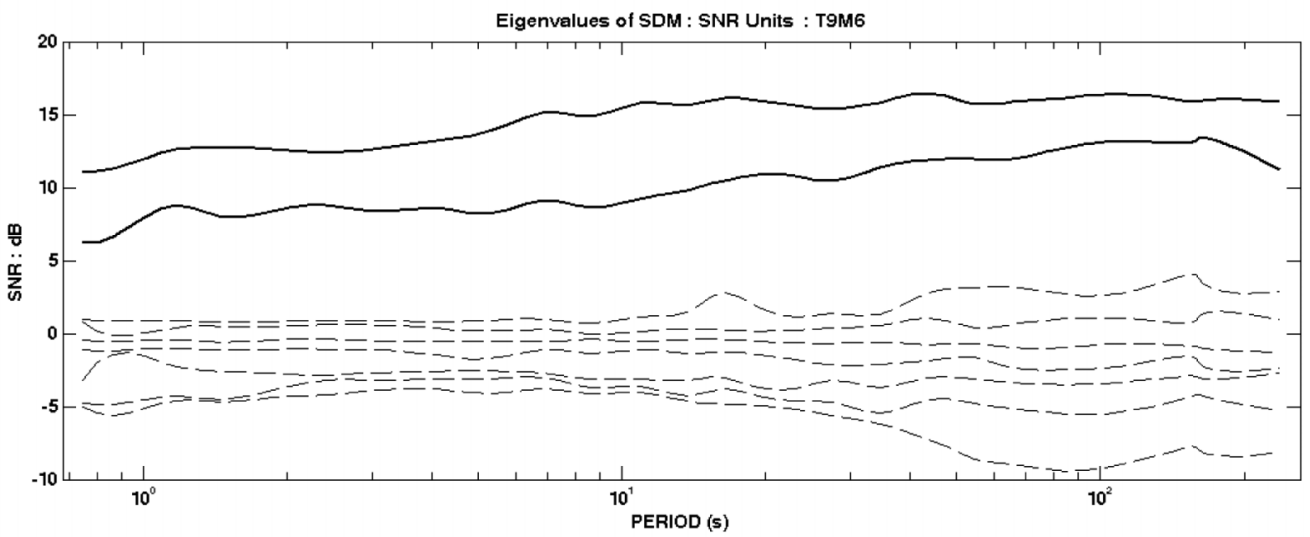

Fig. 7d. Eigenvalues of SDM for TR site: the two dominant eigenvalues are plotted with solid line.

riods. Such results are totally compatible with what emerged analyzing the variograms. A possible explanation for the divergences between the curves, in period range $1-10 \mathrm{~s}$, can be the presence of the «dead band»; the instability past 60 s can be due to shortness of the sets used to estimate the apparent resistivity.

The zrr and zss curves are within the error bars of the characteristic apparent resistivity curves $\left(\rho_{C}\right)$ highlighting the goodness estimates.

Figure $6 \mathrm{c}$ plots the eigenvalues obtained by multivariate analysis of the spectral density matrix as a function of period (SDM, Egbert, 1997). The number of eigenvalues significantly greater than one are two thus effectively confirming of the absence of coherent noise in our MT data.

A similar discussion can be extended to fig. 7a-d in which the apparent resistivity curves in TR site have been computed using MN site as a reference. In this site, the $z r r$ and zss- $x y$ components are well-matched while in $y x$ component the agreement is confined in middle period range. The signal to noise ratio is generally great (fig. 7d).

\section{Conclusions}

Using data from two magnetotelluric stations operating in Agri Valley (Southern Italy) we have computed the apparent resistivity curves for each day divided into intervals of 2 , $4,6,8 \mathrm{~h}$ in a 5 month period (July-November
2006). This segmentation provides the opportunity for a detailed and reliable statistics and establishes the shortest length of the subset to obtain stable apparent resistivity curves. During our investigation period some data segments were contaminated by significant instrument malfunction. To automatically down-weight or eliminate poor quality data a weighted median procedure was applied. The weighted median procedure applied to apparent resistivity values for each frequency and for all subset gives the «characteristic apparent resistivity curve». The characteristic apparent resistivity curves estimated for Marsico Nuovo and Tramutola site can be used to evaluate possible temporal variations in subsoil that could be connected to «seismic stress». The good quality of our data (restricted at high frequency as the apparent resistivity curves are unstable) is also highlighted by eingevalues analysis which supports quasiuniform plane wave MT source assumption without coherent noise. Moreover, comparing the $z r r$ estimates, the single station estimates (zss curve) and $\rho_{C}$ curves we can conclude that the noise involving the Agri Valley is low enough to consider this area reliable for our monitoring study. We can also assess that our weighted median values are good estimators. The sporadic difference between $\rho_{C}$ and $z r r$ curves, in fact, can probably be ascribed to unsatisfactory statistic sample. Expanding the use of the remote reference on longer data set will 
allow us to improve the stability of estimates and to have a continuous indication of the presence/absence of noise and its characterization.

We can conclude that our magnetotelluric estimates are reliable which is one of the key points for the use of apparent resistivity variations as a seismic precursor.

\section{REFERENCES}

BAHR, K. (1988): Interpretation of the magnetotelluric impedance tensor: regional induction and local telluric distortion, J. Geophys., 62, 119-127.

Balasco, M., G. Colangelo, V. Lapenna, M. Loddo, A. SinISCALCHI and L. TelESCA (2004a): Measuring apparent resistivity in a seismically active area of southern Italy, Phys. Chem. Earth, 29, 329-337.

Balasco, M., V. Lapenna, A. Siniscalchi and L. TelesCa (2004b): Stability analysis of apparent resistivity measurement in the seismically active area of Val d'Agri (Southern Italy), Nat. Haz. Earth Sys. Sci., 4, 1-7.

Balasco, M., V. LaPenNa, G. Romano, A. SinisCalchi and L. TELESCA (2007): Extracting quantitative dynamics in Earth's apparent resistivity time series by using the detrended fluctuation analysis, Physica A, 374, 380-388.

CAGNIARD, L. (1953): Basic theory of the magnetotelluric method of geophysical prospecting, Geophysics, 18, 605.

EGBERT, G.D. (1997): Robust multiple-station magnetotelluric data processing, Geophys J. Int., 130, 475-496.

EGBerT, G.D. and J.R. BOOKER (1986): Robust estimation of geomagnetic transfer functions, Geophys. J. R. Astron. Soc., 87, 173-194.

Eisel, M. and G.D. EGBERT (2001): On the stability of magnetotelluric transfer function estimates and the reliability of their variances, Geophys. J. Int., 144, 65-82.
Gamble, T.D., W.M. Goubau and J. Clarke (1979) Magnetotellurics with a remote reference, Geophysics, $\mathbf{4 4}$, 53-68.

Garcia, X. and A.G. Jones (2002): Extended decomposition of MT data in three-dimensional electromagnetics, Methods Geochem. Geophys., 35, 235-250.

Giano, I.G., L. Maschio, M.Alessio, L. Ferranti, S. IMPROTA and M. SCHIATTARELLA (2000): Radiocarbon dating of active faulting in the Agri high valley, Southern Italy, J. Geodyn., 29, 371-386.

HuANG, Q. and T. LiU (2006): Earthquakes and tide response of geoelectric potential field at the Niijima station, Chinese J. Geophys., 49, 1745-1754.

Karakelian, D., G.C. Beroza, S.L. Klemperer and A.C. FRASER-SMITH (2002): Analysis of ultralow-frequency electromagnetic field measurements associated with the 1999 M 7.1 Hector Mine, California, earthquake sequence, Bull. Seismol. Soc. Am., 92 (4), 1513-1524.

Kaufman, A. and G.V. Keller (1981): The magnetotelluric sounding method, Methods Geochem. Geophys., 15, pp. 583.

KelleR, G.V. and F.C. FrischKnecht (1966): Electrical Methods in Geophysical Prospecting (Pergamon Press, New York).

Merzer, M. and S.L. KLemplerer (1997): Modeling lowfrequency magnetic-field precursors to the Loma Prieta earthquake with a precursory increase in fault-zone conductivity, Pure Appl. Geophys., 150, 217-248.

Nagao, T.,Y. Enomoto, Y. Fujinawa, M. Hata, M. HayaKawa, Q. Huang, J. Izutsu, Y. Kushida, K. MaEDA, K. OiKe, S. UYedA and T. Yoshino (2002): Electromagnetic anomalies associated with 1995 Kobe earthquake, J. Geodyn., 33, 401-411.

PARK, S.K., M.J.S. JoHnSton, T.R. MAdDEN, F.D. Morgan and H.F. MORRISON (1993): Electromagnetic precursors to earthquakes in the ULF band; a review of observations and mechanism, Rev. Geophys., 31, 117-132.

ZHAO, Y. and F. QIAN (1994): Geoelectric precursors to strong earthquakes in China, Tectonophysics, 233, 99-113. 